\title{
Die Zukunft von Europol
}

\author{
Michael Niemeier*
}

„Auch wenn ich die Ziele des großen Gesamteuropas begrenze, muss ich die innere Sicherheit auf jeden Fall dabei haben. Denn es ist heutzutage unmöglich, auf diesem Gebiet ohne schlagkräftige Zusammenarbeit zu existieren, da die Kriminalität international agiert und wir alle unter denselben Übeln leiden. "

Die Ausführungen des früheren Kommissionspräsidenten Jacques Delors aus den „Erinnerungen eines Europäers“, die den folgenden Überlegungen vorangestellt sind, treffen zu: Die Gewährleistung von Sicherheit für die Bürgerinnen und Bürger in den Staaten der Europäischen Union kann nicht mehr allein durch nationale, auf der eigenen Souveränität beruhende Maßnahmen realisiert werden. Früh wurde das bereits im Rahmen der Diskussion über die Freizügigkeit in Europa erkannt. Das Ergebnis dieser Diskussion und die Erkenntnis der Notwendigkeit einer engen Zusammenarbeit fanden Niederschlag im Schengener Übereinkommen. Maßnahmen wie die Intensivierung des polizeilichen Informationsaustausches, grenzüberschreitende Observation und Nacheile, Verbesserung der Rechtshilfe, einheitliche Außengrenzkontrollen und das Schengener Informationssystem (SIS) brachten hier erhebliche Verbesserungen.

Mit dem Europäischen Polizeiamt Europol wurde in den 1990er Jahren ein weiteres wirksames Instrument geschaffen: eine europäische Plattform zum Austausch von Informationen, die der Verhinderung und Verfolgung schwerer grenzüberschreitender Kriminalität dienen, und zur Koordinierung entsprechender Maßnahmen der nationalen Strafverfolgungsbehörden. Anhand von Datenzulieferungen der Mitgliedstaaten, aber auch gestuitzt auf die Zusammenarbeit mit Drittstaaten und internationalen Einrichtungen sollen bei Europol Analysen erstellt werden, auf deren Grundlage die nationalen Polizeibehörden dann weitere Maßnahmen ergreifen können. Europol nahm seine Tätigkeiten in vollem Umfang am 1. Juli 1999 auf, nachdem das Europol-Übereinkommen am 1. Oktober 1998 in Kraft getreten war. Dieses hier sehr vereinfacht dargestellte Grundkonzept macht den Mehrwert einer europäischen (Polizei-)Zusammenarbeit sichtbar. Denn die Zusammenfassung von Einzelinformationen ergibt naturgemäß mehr als ihre zahlenmäßige Summe. Durch eine analytische Gesamtschau, das Herstellen von Querverbindungen und Verknüpfungen, schafft sie neue Erkenntnisse und ermöglicht so ein qualitativ verbessertes Vorgehen und damit größere Erfolge im Kampf gegen die Kriminalität. Die europäische Ebene ersetzt also nicht die nationale Verbrechensbekämpfung, sie optimiert sie und macht ihr Handeln effizienter.

Die Frage nach einer künftigen, möglicherweise erweiterten Rolle Europols lässt sich nicht beantworten ohne einen Blick auf die Entstehungsgeschichte der Institution und ihre gegenwärtige Rolle im Rahmen der Kriminalitätsbekämpfung auf europäischer Ebene. Die folgenden Überlegungen gliedern sich daher in drei Schritte:

Zunächst geht es um die Analyse der programmatischen Entwicklung vom Beschluss des Europäischen Rates von Maastricht aus dem Jahr $1991^{2}$ über Tampere ${ }^{3}$ und das Haager Pro-

\footnotetext{
Ministerialrat Michael Niemeier, Referatsleiter im Bundesministerium des Innern, Berlin. Der Artikel beruht auf einem Vortrag vor der Europäischen Rechtsakademie (ERA), Trier, am 17. April 2007 und gibt die persönliche Meinung des Autors wieder.

1 Jacques Delors: Erinnerungen eines Europäers, Berlin 2004, S. 512-513.

2 Vgl. Presseerklärung zu den Schlussfolgerungen des Europäischen Rates in Maastricht, 9./10. Dezember 1991, DOC/91/7 vom 11.12.1991, S. 2 sowie Gemeinsame Maßnahme vom 10. März 1995 bezüglich der EuropolDrogenstelle, vom Rat aufgrund von Artikel K.3 des Vertrags über die Europäische Union beschlossen, 95/73/ JI, in: Amtsblatt der EU, Nr. L 062/1995, S. 1.

3 Europäischer Rat (Tampere): Schlussfolgerungen des Vorsitzes, 15./16. Oktober 1999, Nr. 200/1/99, RS 43 und 55.
} 
gramm ${ }^{4}$ bis hin zum Programm der deutschen EU-Ratspräsidentschaft. ${ }^{5}$ Der zweite Teil befasst sich mit den gegenwärtigen Herausforderungen, die sowohl im Bereich bestimmter Deliktsformen als auch strukturell im institutionellen Gefüge einer ,Europäischen Sicherheitsarchitektur' liegen. Schließlich wird drittens nach künftigen Perspektiven für Europol gefragt, bei denen weder die Zukunft des Verfassungsvertrages noch eine langfristig wohl unvermeidliche stärkere Zentralisierung der Gewährleistung der Sicherheit in Europa außer Acht gelassen werden können.

\section{Programmatische Entwicklung}

Die Gründung Europols geht auf den Beschluss des Europäischen Rates von Maastricht im Dezember 1991 zurück. Dieser erwähnt Europol und seine Ausgangsaufgabe, die Organisation des europäischen Informationsaustauschs im Bereich der Drogenbekämpfung, bloß in zwei Sätzen. Zuvor war jedoch auf dem Juni-Gipfel in Luxemburg ein Ausbau der Aufgabenwahrnehmung Europols in zwei Phasen ins Auge gefasst worden: In der ersten Phase sollte eine ,Relaisstelle“ für den wechselseitigen Austausch von Informationen und Erfahrungen eingerichtet werden; die zweite Phase sah hingegen (bereits 1991!) die Einräumung konkreter Handlungsbefugnisse auch in den Mitgliedstaaten vor (,,powers to act also within the Member States"). ${ }^{6}$

\section{Der Rat von Tampere}

Acht Jahre später, in den Schlussfolgerungen zum Europäischen Rat von Tampere im Jahre 1999, ${ }^{7}$ ist von operativen Befugnissen auf dem Gebiet der Mitgliedstaaten nicht mehr die Rede. Zwar wird Europol ausdrücklich eine Schlüsselrolle bei der Kriminalitätsbekämpfung in Europa zuerkannt. Im Einzelnen gefordert werden jedoch (lediglich) Maßnahmen wie die Einrichtung gemeinsamer Ermittlungsteams der Mitgliedstaaten, an denen Europol sich ,,in unterstützender Funktion beteiligen“ soll, die Möglichkeit Europols, einzelne Mitgliedstaaten um die Einleitung von Ermittlungen zu ersuchen oder eine von der Art der Vortaten unabhängige Ausweitung der Zuständigkeit Europols auf den Bereich der Geldwäsche.

In einer im Oktober 2001 erfolgten Bewertung der Schlussfolgerungen von Tampere durch den damaligen belgischen Ratsvorsitz werden Defizite gerade im Kernbereich der Arbeit von Europol konstatiert, nämlich bei der informationsgestützten Analysetätigkeit. Zurückgeführt wird dies in erster Linie auf eine ,übertriebene Zurückhaltung der Mitgliedstaaten bei der Übermittlung der in ihrem Besitz befindlichen sensiblen Informationen an Europol“" ${ }^{8}$ Eine Beurteilung, die auch heute, sechs Jahre später, nicht realitätsfern ist.

\section{Das Haager Programm}

Auch die Beschlüsse zum Haager Programm bedeuten für Europol keinen wirklichen qualitativen Fortschritt. ${ }^{9}$ So fordert der Europäische Rat die Mitgliedstaaten nachdrücklich

4 Haager Programm zur Stärkung von Freiheit, Sicherheit und Recht in der Europäischen Union, in: Amtsblatt der EU, Nr. C 53/2005, S. 1-4.

5 Bundesministerium des Innern: Europa sicher leben. Arbeitsprogramm des Bundesministeriums des Innern für die deutsche EU-Ratspräsidentschaft im ersten Halbjahr 2007, abrufbar unter: http://www.eu2007.bmi.bund.de (letzter Zugriff: 29. Mai 2007).

6 Vgl. Presseerklärung zu den Schlussfolgerungen des Europäischen Rates in Luxemburg, 28./29. Juni 1991, DOC/91/2 vom 29.6.1991, S. 6.

7 Europäischer Rat (Tampere): Schlussfolgerungen des Vorsitzes, 15./16. Oktober 1999.

8 Vgl. Aufzeichnung des Vorsitzes, Dokument 13416/01 JAI 133 vom 30.10.2001, S. 8.

9 Haager Programm, 2005. 
auf, Europol in die Lage zu versetzen, eine entscheidende Rolle bei der Bekämpfung der Schwerkriminalität zu übernehmen. Europol sollen alle erforderlichen Informationen rechtzeitig zur Verfügung gestellt werden und eine gute Zusammenarbeit zwischen den nationalen Behörden und Europol sei zu fördern. Darüber hinaus werden die Mitgliedstaaten aufgefordert, die noch ausstehenden Änderungsprotokolle bis Ende $2004 \mathrm{zu}$ ratifizieren. Bekanntlich ist diese Ratifizierung für die ersten beiden Protokolle am 28. März und für das dritte Protokoll erst am 18. April 2007 erfolgt. ${ }^{10}$

Einzig neu ist die Entscheidung, dass die Mitgliedstaaten Europol zukünftig zur Zentralstelle der Union für die Bekämpfung der Euro-Fälschung erklären sollen. Dies ist zwischenzeitlich durch einen Ratsbeschluss erfolgt. ${ }^{11}$ Weiter verweist das Haager Programm auf das Inkrafttreten des Entwurfs des Vertrages über eine Verfassung für Europa und sieht vor, dass spätestens zum 1. Januar 2008 gemäß Artikel III-276 des Verfassungsentwurfs ein europäisches Gesetz über Europol erlassen wird. Dabei soll ,allen Europol übertragenen Aufgaben Rechnung" getragen werden. ${ }^{12}$

Leider muss festgestellt werden, dass zwei Jahre nach Beschlussfassung über das Haager Programm in der Umsetzung noch relativ geringe Fortschritte zu verzeichnen sind. Zwar hat der Ministerrat recht schnell die neue Funktion von Europol als Zentralstelle zur Bekämpfung der Euro-Fälschung beschlossen. Diese Funktion ist aber zunächst auf die Annahme von Daten aus Drittstaaten begrenzt. Die Zuständigkeit der nationalen Strafverfolgungsbehörden für die Euro-Fälschung wird dadurch nicht tangiert. Mit der 2006 neu eingeführten jährlichen ,Bewertung der Bedrohungslage“ im Bereich der Organisierten Kriminalität (OCTA $)^{13}$ wurde immerhin die Darstellung der Analyseergebnisse seitens Europols wesentlich verbessert. Die übrigen im Haager Programm genannten praktischen Maßnahmen der Zusammenarbeit zwischen Europol und den Mitgliedstaaten konnten aufgrund der bis vor Kurzem fehlenden Ratifikation der drei Änderungsprotokolle nicht in die Praxis umgesetzt werden.

\section{Das Programm der deutschen EU-Ratspräsidentschaft}

Neben der Schaffung eines europäischen Informationsverbundes ist die Stärkung von Europol einer der Schwerpunkte des Programms der deutschen Ratspräsidentschaft im Bereich der Innenpolitik. ${ }^{14}$ Auf Grundlage der Schlussfolgerungen des Rates zur Zukunft von Europol $^{15}$ soll insbesondere die operative Arbeit von Europol weiter verbessert werden. Dazu ge-

10 Protokoll erstellt aufgrund von Artikel 43 Absatz 1 des Übereinkommens über die Errichtung eines Europäischen Polizeiamts (Europol-Übereinkommen) zur Änderung von Artikel 2 und des Anhangs jenes des Übereinkommens - Erklärung, in: Amtsblatt, Nr. C 358/2000, S. 2-7.

PROTOKOLL - zur Änderung des Übereinkommens über die Errichtung eines Europäischen Polizeiamts (Europol-Übereinkommen) und des Protokolls über die Vorrechte und Immunitäten für Europol, die Mitglieder der Organe, die stellvertretenden Direktoren und die Bediensteten von Europol, in: Amtsblatt, Nr. C 312/2002, S. 2-7.

PROTOKOLL - aufgrund von Artikel 43 Absatz 1 des Übereinkommens über die Errichtung eines Europäischen Polizeiamts (Europol-Übereinkommen) zur Änderung dieses Übereinkommens, in: Amtsblatt, Nr. C 2/ 2004, S. 3-12.

11 Rat der Europäischen Union: Beschluss 2005/511/JI des Rates vom 12. Juli 2005 über den Schutz des Euro gegen Fälschung durch Benennung von Europol als Zentralstelle zur Bekämpfung der Euro-Fälschung, in: Amtsblatt, Nr. L 185/2005, S. 35-36.

12 Haager Programm, 2005, S. 9.

13 Europol: Organized Crime Threat Assessment 2006, abrufbar unter: http://www.europol.eu.int/publications/ OCTA/OCTA2006.pdf (letzter Zugriff: 29. Mai 2007).

14 Bundesministerium des Innern: Europa sicher leben, 2007.

15 Rat der Europäischen Union: Mitteilung an die Presse, 2768. Tagung des Rates Justiz und Inneres, 15801/06 (Presse 341), 4.-5. Dezember 2006 sowie Dok. 14405/4/06 REV 4 EUROPOL 93. 
hört neben einer maßvollen Erweiterung des Zuständigkeitsbereichs auch eine Entbürokratisierung und Flexibilisierung der Regelungen und Abläufe bei Europol.

\section{Operative Verbesserungen}

Zukünftig soll die Zuständigkeit von Europol auch auf die Verhütung und Bekämpfung von Delikten der nicht organisierten schweren grenzüberschreitenden Kriminalität erweitert werden. Auf diese Weise könnte Europol die Mitgliedstaaten künftig auch bei der Verfolgung von Straftaten durch reisende Gewalttäter und Hooligans, grenzüberschreitend tätige Serienmörder oder der Verbreitung von Kinderpornografie im Internet unterstützen. Damit sollen vor allem Abgrenzungsschwierigkeiten hinsichtlich der Zuständigkeit von Europol beseitigt werden.

Im Hinblick auf Europol spielt schließlich auch der Ausbau des europäischen Informationsverbundes als Ziel der deutschen Präsidentschaft eine wichtige Rolle. Der Informationsverbund bezweckt die künftige Gewährung umfassenden grenzüberschreitenden Zugriffs auch auf die für die nationalen Polizeien verfügbaren Daten. In einem ersten Schritt wird auf der Grundlage des Prümer Vertrages ${ }^{16}$ zu bestimmten Datenkategorien ein solcher Zugriff im sogenannten Hit-/no-hit-Verfahren gewährleistet. Auch die Rolle von Europol in diesem Informationsverbund muss geklärt werden. Während Europol im Rahmen der Fortentwicklung des SIS zukünftig beispielsweise Zugang zu den dort gespeicherten Daten erhält, ist das bei den nationalen Datenbanken bis jetzt nicht der Fall. Hier ist zu überlegen, inwieweit Europol (und auch der europäischen Service-Einheit für justizielle Zusammenarbeit Eurojust) ein Zugang zu (gemeinsamen) Informationssystemen ermöglicht werden soll, soweit dies zur Erfüllung ihrer Aufgaben erforderlich ist.

\section{Entbürokratisierung / Flexibilisierung}

Hinsichtlich der Verfahrensregelungen und internen Abläufe von Europol wird es erforderlich sein, die vorhandenen Regelungen nochmals kritisch zu überprüfen. Dazu gehört auch die Vielzahl von Vorlagepflichten an den Rat der Justiz- und Innenminister. Hier sollte überlegt werden, ob nicht in einigen Fällen der Europol-Verwaltungsrat ${ }^{17}$ abschließend entscheiden kann. Auch sollten in diesem Zusammenhang die Regeln des internen Managements und des Personaleinsatzes überprüft werden. Sie haben sich teilweise als nicht flexibel genug erwiesen. So kann beispielsweise auf neue operative Prioritäten nicht in ausreichendem Maß durch Personalverschiebung innerhalb der Organisation reagiert werden.

Ein weiterer Punkt in diesem Zusammenhang betrifft die Arbeit des Verwaltungsrats von Europol. Nach derzeit verbindlicher Praxis ist der Vorsitz im Europol-Verwaltungsrat an die jeweilige Ratspräsidentschaft gekoppelt. Das bedeutet einen halbjährlichen Wechsel des Vorsitzes. Eine solche hohe Diskontinuität ist der Arbeit eines Gremiums, das neben administrativen Vorgängen auch die strategische wie operative Ausrichtung Europols kontinuierlich begleiten soll, nicht förderlich. Es sollte deshalb in Betracht gezogen werden, den Vorsitz des Verwaltungsrats unabhängig von der jeweiligen EU-Ratspräsidentschaft für einen Zeitraum von zwei Jahren zu wählen, der beispielsweise um weitere zwei Jahre verlängert

16 Vertrag zwischen dem Königreich Belgien, der Bundesrepublik Deutschland, dem Königreich Spanien, der Französischen Republik, dem Großherzogtum Luxemburg, dem Königreich der Niederlande und der Republik Österreich über die Vertiefung der grenzüberschreitenden Zusammenarbeit, insbesondere zur Bekämpfung des Terrorismus, der grenzüberschreitenden Kriminalität und der illegalen Migration, Prüm am 27. Mai 2005, abrufbar unter: http://www.bmj.bund.de/files/-/974/Prümer_Vertrag.pdf (letzter Zugriff: 29. Mai 2007).

17 Der Verwaltungsrat von Europol besteht aus je einem Vertreter pro EU-Mitgliedstaat und nimmt unterschiedliche Kontroll- und Managementaufgaben gemäß Artikel 28 des Europol-Übereinkommens wahr. 
werden könnte. Ebenfalls begrenzt werden könnte die Amtszeit der Delegierten im Verwaltungsrat. Entsprechende Modelle werden bei anderen Agenturen wie etwa bei der europäischen Agentur für die operative Zusammenarbeit an den Außengrenzen Frontex bereits praktiziert und ließen sich für Europol leicht übernehmen.

Schließlich könnte auch die Rolle der für Europol zuständigen nationalen Stellen in der jetzigen Gremienstruktur weiter ausgebaut werden. Europol ,lebt' gewissermaßen von der Zusammenarbeit mit und Verankerung in den Polizeibehörden der Mitgliedstaaten. Eine Schlüsselstellung kommt dabei den Leitern der nationalen Stellen (Heads of National Units - HENUs) zu, die als Bindeglied in beide Richtungen wirken. Daher sollte die Idee eines hochrangig besetzten Gremiums von Repräsentanten der nationalen Stellen (sogenannte ,Super-HENUs') geprüft werden, das Europol und insbesondere dem Verwaltungsrat vor allem in Fragen von operativer Bedeutung als echtes, regelmäßig tagendes Beratergremium zur Seite stehen könnte.

\section{Herausforderungen}

Die Strafverfolgungsbehörden der Mitgliedstaaten der Europäischen Union und damit auch Europol sind gegenwärtig mit erheblichen Herausforderungen konfrontiert, die sowohl im Bereich einzelner Kriminalitätsphänomene liegen als auch den institutionellen Rahmen der Sicherheitsarchitektur einer auf 27 Mitgliedstaaten erweiterten Europäischen Union betreffen.

\section{Kriminalitätsbezogene Herausforderungen}

Eines der bedrohlichsten Phänomene für Europa wie für die gesamte Völkergemeinschaft ist ohne Frage der islamistische Terrorismus. Die Herkunft seiner Täter, ihre Aktionsgebiete und ihre Ziele sind weder regional noch national, ja nicht einmal auf einen Kontinent beschränkt. Der islamistische Terrorismus gefährdet weltweit Sicherheit und Stabilität und hat längst auch Europa zu einem Teil des allgemeinen Gefahrenraums gemacht. Die Terroristen rekrutieren sich aus allen Teilen der islamischen Welt wie auch aus in Europa lebenden Unterstützern. Sie eint das gemeinsame Feindbild und die ideologische Verbundenheit. Es besteht keine straffe Kaderorganisation, sondern ein Geflecht verschiedener, selbständig und damit hochgradig flexibel agierender Gruppierungen und Zellen.

Nicht zu vernachlässigen sind daneben, worauf auch der jüngste Terrorismusbericht von Europol ${ }^{18}$ hinweist, ethno-nationalistische und separatistische Formen des Terrorismus sowie Links- und Rechtsterrorismus. Gruppierungen, die diesem Spektrum zuzuordnen sind, haben in der Europäischen Union im Jahr 2006 zusammengerechnet rund 480 Anschläge verübt. Regional bedingte Konflikte bleiben dabei nicht auf ihren Ursprungsort beschränkt, sondern breiten sich mit Hilfe moderner Kommunikationsmittel sowie über Migrationsbewegungen auch in die europäische Lebenswelt aus. Wenn junge Männer aus dem Nahen Osten mit einer radikal-islamischen Vorprägung zum Studium nach Deutschland kommen und dort Kofferbomben in Zügen platzieren, weil in Dänemark Karikaturen des Propheten Mohammed publiziert wurden, zeigt dies drastisch die Entkoppelung von Ursachen und Austragungsorten entsprechender Konflikte.

Als zweiter wesentlicher Kriminalitätsbereich neben dem Terrorismus ist die Organisierte Kriminalität zu nennen. Auch hier schreitet die Internationalisierung in großen Schrit-

18 Europol: EU Terrorism Situation and Trend Report 2007, abrufbar unter: http://www.europol.europa.eu/publications/TESAT/TESAT2007.pdf (letzter Zugriff: 29. Mai 2007). 
ten voran. In Deutschland werden jährlich über 600 Ermittlungsverfahren bearbeitet, die der Organisierten Kriminalität zuzuordnen sind. Die Zahl der Tatverdächtigen liegt jährlich bei mehr als 10.000. Dass in 77 Prozent der Verfahren Tatverdächtige unterschiedlicher Nationalität festgestellt worden sind, deutet darauf hin, dass sich auch hier tradierte, ethnisch oder in sonstiger Weise homogen kodierte Gruppierungen neu formieren. Wesentliche Deliktschwerpunkte im Bereich der Organisierten Kriminalität sind Rauschgifthandel und Rauschgiftschmuggel, Eigentumskriminalität, Schleuserkriminalität, Menschenhandel, Steuer- und Zolldelikte, Gewaltkriminalität, Fälschungskriminalität, Waffenhandel und Waffenschmuggel und Umweltkriminalität. Mit einem Anteil von über 30 Prozent nimmt die Rauschgiftkriminalität eine herausragende Position ein. Die ermittelten Schadenshöhen und Gewinne variieren von Jahr zu Jahr erheblich. Im Jahr 2005 wurde ein Schaden in Höhe von circa 688 Mio. Euro und ein Gewinn in Höhe von circa 842 Mio. Euro festgestellt. ${ }^{19}$ Die Dunkelziffer dürfte deutlich darüber liegen. Das starke Wohlstandsgefälle innerhalb Europas und gegenüber anderen Teilen der Welt verbunden mit zunehmenden Globalisierungs- und Freizügigkeitstendenzen ist geeignet, diese Entwicklung weiter zu forcieren. Zugleich kommt damit der grenzüberschreitenden Zusammenarbeit im Kampf gegen die (internationale) Organisierte Kriminalität ein immer größerer Stellenwert zu. Rein auf die nationale Ebene ausgerichtete Bekämpfungsansätze der Organisierten Kriminalität werden zunehmend an Bedeutung verlieren.

\section{Institutionelle Herausforderungen}

Durch die Erweiterung der Europäischen Union auf nun 27 Mitgliedstaaten erhöht sich sowohl das Informationsaufkommen als auch der Informationsbedarf der Strafverfolgungsbehörden erheblich. Die Mobilität von Tätern in der Europäischen Union wird durch den Wegfall der Binnengrenzkontrollen weiter zunehmen. Gesteigerte Anforderungen an Europol sowohl als Informationsvermittler wie auch im Analysebereich werden die Folge sein. Dies wird eine deutliche Ausweitung der (informations-)technischen Kapazitäten von Europol einschließen.

Mit dem im Haager Programm beschlossenen Grundsatz der Verfügbarkeit stellt sich auch für Europol die Frage, wie es in den zunehmenden Informationsaustausch zwischen den Mitgliedstaaten einbezogen wird. Dies betrifft nicht nur den bi- und multilateralen Austausch, sondern auch den Zugang zu europäischen Datenbanken wie dem SIS, dem VisaInformationssystem (VIS) und auch zu Eurodac, dem gemeinschaftsweiten System zur Abnahme der Fingerabdrücke von Asylbewerbern.

Im Rahmen der europäischen Sicherheitsarchitektur ist Europol nicht mehr die einzige auf europäischer Ebene gegründete Institution für die Verhinderung und Bekämpfung schwererer grenzüberschreitender Kriminalität. Mit Einrichtungen wie dem Europäischen Amt für Betrugsbekämpfung (OLAF), Eurojust, dem gemeinsamen Lagezentrum (SitCen) und jüngst Frontex sind weitere Akteure hinzugetreten. Partielle Zuständigkeitsüberschneidungen wie etwa zwischen Europol und Frontex im Bereich der illegalen Migration weisen darauf hin, dass es künftig eine einheitliche und eindeutige Konzeption für die Aufgabenwahrnehmung der europäischen Sicherheitsbehörden geben muss. Sollte dies nicht gelingen, wird zunehmend mit Doppelarbeit und daraus resultierenden Ineffizienzen zu rechnen sein, die der Sicherheitslage in Europa nicht zuträglich sind.

19 Vgl. Bundeskriminalamt: Bundeslagebild Organisierte Kriminalität 2005, Pressefreie Kurzfassung, Wiesbaden, Juli 2006. 
Ein letzter Aspekt der institutionellen Herausforderungen betrifft die parlamentarische und gerichtliche Kontrolle von Europol. ${ }^{20}$ Seit vielen Jahren fordern das Europäische Parlament und auch nationale Parlamente, dass beide Aspekte deutlich zu verbessern seien. Bemängelt wird vor allem ein Defizit der parlamentarischen Kontrolle auf europäischer Ebene. Die nationale Kontrolle durch die Mitgliedstaaten sei für eine europäische Institution nicht ausreichend. Am 10. April 2007 wurde in einer Anhörung des Ausschusses für bürgerliche Freiheiten, Justiz und Inneres des Europäischen Parlaments zu Europol von den dort vertretenen Abgeordneten erneut die Idee eines gemeinsamen Ausschusses mit Vertretern nationaler Parlamente und des Europäischen Parlaments zur Kontrolle von Europol vorgeschlagen. Daneben wird auch immer wieder eine stärkere gerichtliche Kontrolle gefordert.

\section{Perspektiven}

Die nächsten Schritte der Entwicklung Europols sind durch das Inkrafttreten der drei Änderungsprotokolle zur Europolkonvention unmittelbar vorgezeichnet. ${ }^{21}$

Die operative Arbeit des Europäischen Polizeiamts wird spätestens mit dem Inkrafttreten der letzten Instrumente zur Umsetzung der Änderungsprotokolle in vier Bereichen eine wesentliche Verbesserung erfahren:

- Durch die Teilnahme an gemeinsamen Ermittlungsteams wird Europol zukünftig unmittelbar Daten aus den Analysedateien (AWFs) in die Arbeit der Ermittlungsteams einbringen können. Dieser unmittelbare Datenaustausch wird zu einer spürbaren Verbesserung der Qualität der in den AWFs gespeicherten Informationen und damit zu ihrer noch praxisgerechteren Ausrichtung führen.

- Die Ersuchenskompetenz von Europol schafft die Möglichkeit, unmittelbar aus der Analysetätigkeit heraus auf die Mitgliedstaaten zuzugehen und dort weitere Ermittlungen anzuregen. Damit kann Europol aus seiner bisher eher passiven Rolle als Informationsvermittler verstärkt heraustreten und nun auch eine aktive Rolle spielen. Die Akzeptanz dieser neuen Rolle bei den Strafverfolgungsbehörden der Mitgliedstaaten wird maßgeblich von der Qualität der dem jeweiligen Ersuchen zugrunde liegenden Analyse abhängen.

- Mit der Erweiterung der Analyseteams auf Teilnehmer aus Drittstaaten und Drittstellen wird die Arbeit der Teams selbst verbessert. Die Regelung wird insbesondere zu einer Erweiterung der Informationsbasis der AWFs führen, da Drittstaaten und -stellen eher bereit sein werden, sensible Informationen zu übermitteln, wenn sie mit eigenem Personal an der Auswertung beteiligt sind.

- Neben den für Europol zuständigen nationalen Stellen erhalten zukünftig weitere Stellen der Mitgliedstaaten die Möglichkeit, selbst auf das Europol-Informationssystem zuzugreifen. Allerdings ist dieser direkte Zugriff auf ein sogenanntes Hit-/no-hit-Verfahren begrenzt. Die vollständige Information muss sodann über die nationale Stelle eingeholt werden.

Eine schnelle tatsächliche Umsetzung dieser Maßnahmen in den Mitgliedstaaten ist notwendig, damit die mit ihnen bezweckten Erfolge zeitnah realisiert werden können. Neben diesen kurzfristigen Verbesserungen der Arbeit von Europol werden sich weitere Fortschritte durch die unter deutscher EU-Ratspräsidentschaft begonnene Umwandlung der Europol-Konvention in einen Ratsbeschluss ergeben. Dies gilt insbesondere für die im

20 Im Einzelnen Jan Ulrich Ellermann: Europol und FBI, Baden-Baden 2005, S. 319-409.

21 Protokolle zur Änderung des Europol-Übereinkommens von 2000 und 2002, in Kraft seit 29. März 2007 sowie das Protokoll zur Änderung des Europol-Übereinkommens von 2003, in Kraft seit 18. April 2007. 
Beschlussentwurf vorgesehene Zuständigkeitserweiterung auf alle Formen grenzüberschreitender Schwerkriminalität wie auch für administrativ-verfahrensmäßige Verbesserungen, so zum Beispiel die ebenfalls bereits erwähnte Straffung der Arbeit des Verwaltungsrats oder die Rolle der Leiter der nationalen Stellen.

\section{Reformvorschläge und Bestimmungen des Verfassungsvertrages}

Wie wird sich, wie soll sich Europol darüber hinaus zukünftig weiterentwickeln? Mit dieser Frage hat sich auch der Europäische Konvent zur Erarbeitung einer Europäischen Verfassung beschäftigt, auf dessen Arbeiten hier kurz einzugehen ist. ${ }^{22}$ In der zuständigen Arbeitsgruppe X: Freiheit, Sicherheit und Recht des Europäischen Konvents war eines der Schwerpunktthemen die Stärkung der operativen Zusammenarbeit in Europa. Dabei stellte die Arbeitsgruppe fest, dass die operative Zusammenarbeit ,derzeit nicht effizient, transparent und zuverlässig genug“ sei. ${ }^{23}$ Neben einer stärkeren Trennung der legislativen von der operativen Arbeit im Rat wurde insbesondere der Ausbau von Europol und Eurojust gefordert. Statt einer detaillierten Beschreibung der Zuständigkeiten sollte zukünftig eine allgemeine Regelung dem Gesetzgeber größere Spielräume geben. Es sollte auf die zentrale Rolle von Europol im Rahmen der polizeilichen Zusammenarbeit in Europa hingewiesen werden. Eine Erweiterung der Zuständigkeit auf alle schweren Formen der grenzüberschreitenden Kriminalität wird vorgeschlagen. Auch die Koordinierung und Durchführung von Ermittlungen sowie die Mitwirkung an operativen Maßnahmen, die gemeinsam mit den Behörden der Mitgliedstaaten durchzuführen sind, wäre Inhalt der vom Gesetzgeber zu treffenden Regelung. Als Kompetenzbegrenzung sei vorzusehen, dass Europol nur in Verbindung und Absprache mit dem betreffenden Mitgliedstaat auf dessen Gebiet operativ tätig werden dürfe und Zwangmaßnahmen den Beamten des Mitgliedstaates vorbehalten bleiben.

Dieser Vorschlag entsprang auch der Einsicht, dass die bisherigen Regelungen zu Europol in den Verträgen eher den politischen Rahmen vorgaben. Sobald dieser ausgefüllt ist (so Artikel 30 Absatz 2 lit. a. und b. EU-Vertrag), hatte sich der Regelungsinhalt erschöpft. Eine allgemeine Kompetenznorm dagegen ist offen und lässt dem Gesetzgeber im vorgegebenen Rahmen mehr Möglichkeiten. Die von der Arbeitsgruppe X vorgeschlagene Begrenzung ist dann auch in den Artikel III-276 des Verfassungsentwurfs übernommen worden.

Was hieße das nun für die Kompetenzen von Europol? Europol hätte im Falle der Übernahme dieser Regelung zukünftig die Zuständigkeit, die Tätigkeit der Polizeibehörden und anderen Strafverfolgungsbehörden der Mitgliedstaaten bei der Verhütung und Bekämpfung schwerer Kriminalität zu unterstützen und zu verstärken.

Näher konkretisiert wird der Auftrag durch Absatz 2 des Artikels III-276 Verfassungsentwurf. Zu den Aufgaben von Europol kann danach die Datenverarbeitung und die Koordinierung, Organisation und Durchführung von Ermittlungen und von operativen Maßnahmen gehören. Ermittlungen und operative Maßnahmen können nur gemeinsam mit den zuständigen Behörden der Mitgliedstaaten oder im Rahmen von gemeinsamen Ermittlungsteams durchgeführt werden. Was würde das konkret bedeuten?

Im Bereich der Datenverarbeitung wäre durchaus vorstellbar, dass Europol über die beiden bisherigen Systeme (Analysedateien und Informationssystem) hinaus auch Fahndungsdateien einrichtet. So gab es bereits 2003 den Vorschlag aus dem Kreis der sogenannten G-6-Staaten (Deutschland, Frankreich, Großbritannien, Italien, Spanien und

22 Ausführlich Georg Srock: Rechtliche Rahmenbedingungen für die Weiterentwicklung von Europol, Tübingen 2006, S. 203-252.

23 Europäischer Konvent: Schlussbericht der Gruppe X „Freiheit, Sicherheit und Recht“, CONV 426/02, S. 15. 
Polen), das SIS durch Europol verwalten zu lassen. Allerdings wäre dies damals rechtlich wohl nur durch eine Änderung der Europol-Konvention möglich gewesen. Darüber hinaus wäre auch denkbar, dass ein europaweites Fingerabdrucksystem in Zukunft durch Europol geführt wird.

\section{Offene Fragen}

Hinsichtlich der Koordinierung, Organisation und Durchführung von Ermittlungen und von operativen Maßnahmen bleibt der Kompetenzrahmen nach den Maßgaben des Verfassungsvertrags unklar. Wie soll man sich konkret vorstellen, dass Europol Ermittlungen und operative Maßnahmen ,gemeinsam “ mit den zuständigen Behörden durchführt? Unklarheiten hinsichtlich der Verantwortung für eine bestimmte Ermittlungsmaßnahme darf es nicht geben. Welche Rolle könnte Europol dann aber bei den Ermittlungen spielen, die substanziell über die bereits jetzt in Kraft getretene Möglichkeit zur Teilnahme an gemeinsamen Ermittlungsgruppen hinausgeht? Hier wird dem Gesetzgeber und damit der Politik eine erhebliche Kraftanstrengung abverlangt, diese schwierige Frage zufriedenstellend zu lösen.

Unabhängig vom Verfassungsvertrag und seinem derzeit noch unklaren Schicksal wird in der politischen Diskussion immer wieder die Erweiterung der operativen Tätigkeit von Europol gefordert. Soweit dies auch die Durchführung eigenständiger Ermittlungen meint, wird zu Recht auf den fehlenden einheitlichen Rechtsrahmen in der Europäischen Union verwiesen. Das Fehlen eines einheitlichen Straf- und Strafverfahrensrechts macht eine Fortentwicklung Europols in diese Richtung auf absehbare Zeit unmöglich, von der Frage der Akzeptanz durch die Strafverfolgungsbehörden der Mitgliedstaaten einmal völlig abgesehen. Auch müsste das Verhältnis zu Eurojust bei der Durchführung von Ermittlungen neu bestimmt werden. Sollte im Fall eigenständiger Ermittlungen durch Europol nicht Eurojust beziehungsweise eine europäische Staatsanwaltschaft die Verfahrensleitung übernehmen? Damit verbunden ist auch die Frage der Fortgeltung der Immunitätsregelungen für Europolbedienstete und der parlamentarischen und gerichtlichen Kontrolle ihrer Tätigkeit.

\section{Ausblick}

Sowohl die aufgezeigten Herausforderungen als auch die ansatzweise - wie etwa im Entwurf des Verfassungsvertrages - bereits angelegten Perspektiven machen deutlich, dass sich das , $\mathrm{Ob}^{\text {“ }}$ der Frage nach einer künftigen erweiterten Rolle Europols im Raum der Freiheit, der Sicherheit und des Rechts nur mit einem klaren Ja beantworten lässt. Ein Verharren im Status quo oder gar ein Zurückgehen hinter bereits Erreichtes wäre mit der Verpflichtung aller Mitgliedstaaten, die Sicherheit ihrer Bürgerinnen und Bürger optimal zu gewährleisten, unvereinbar.

Im Einzelnen darzulegen, wie diese erweiterte Rolle ausgestaltet werden sollte, bedarf zunächst weiterer Arbeiten der für die jeweiligen Sachbereiche zuständigen Experten und vor allem klarer politischer Entscheidungen. Gleichwohl sollen abschließend - gewissermaBen im Sinne einer ,realistischen Vision“ - einige Eckpunkte benannt werden, die bei dem weiteren Arbeits- und Entscheidungsprozess berücksichtigt werden sollten:

Angesichts des ständig steigenden Internationalisierungsgrades wesentlicher Bereiche der Schwerkriminalität wird die Europäische Union, wie dies der deutsch-französische Vorschlag der Außenminister Fischer und de Villepin aus dem Jahr $2002^{24}$ bereits vorsieht, auf die Schaffung eines ,Europäischen Strafverfolgungsorgans‘ langfristig nicht verzichten können.

24 Vgl. Europäischer Konvent: Deutsch-französische Vorschläge für den Konvent zum Raum der Freiheit, der Sicherheit und des Rechts, vorgelegt von Herrn Joschka Fischer und Herrn Dominique de Villepin, Gruppe X „Freiheit, Sicherheit und Recht“: Arbeitsdokument 32, Brüssel, 27.11.2002. 
Bei der Ausgestaltung dieser Einrichtung sollten vor allem drei Kernbereiche höchste Priorität haben:

- ein modernes Informationsmanagement, das eine ebenso schnelle wie intelligente Sichtung, Verarbeitung und Aktualisierung immer größer werdender Datenmengen erlaubt;

- eine fundierte, computerbasierte Ermittlungsunterstützung, die in der Lage ist, aus den vorhandenen Informationen zielgenaue Analysen und Hinweise zur Förderung komplexer Ermittlungsverfahren zu generieren und nicht zuletzt

- effiziente Strukturen und klare Zuständigkeitsabgrenzungen, die einen flexiblen Ressourceneinsatz ermöglichen und Aufgabenüberschneidungen mit anderen Agenturen vermeiden.

Ein ,Europäisches Strafverfolgungsorgan“ sollte außerdem über einen möglichst breiten Zugang zu vorhandenen, polizeilich relevanten Datensammlungen und Fahndungssystemen verfügen. Auch sollte überlegt werden, inwieweit Europol zukünftig zentral in der Europäischen Union (Fahndungs-)Datenbanken führen sollte (wie beispielsweise das Europäische Fingerabdrucksystem, EU-AFIS).

Auch die immer wieder thematisierte Frage nach eigenen Ermittlungszuständigkeiten wird zu beantworten sein. Originäre Zuständigkeiten im Bereich des Schutzes von Gemeinschaftsgütern erscheinen nahe liegend. Neben Straftaten wie etwa der Euro-Fälschung kämen auch Betrugsdelikte zu Lasten der Gemeinschaft in Betracht. Letzteres würde eine Neubestimmung des Verhältnisses zu OLAF, gegebenenfalls auch eine Fusion beider Einrichtungen erforderlich machen.

Gibt es eine Entscheidung zugunsten solcher Ermittlungskompetenzen, müssten diese schließlich auch staatsanwaltschaftlich adäquat gespiegelt werden. Der Gedanke einer Europäischen Staatsanwaltschaft und eine Harmonisierung des Straf- und Strafverfahrensrechts in Europa werden dabei wesentliche Stichworte sein.

\section{integration}

\section{Neue Online-Artikelsuche}

Anlässlich des 30. Jahrgangs der integration hat die Redaktion ein Online-Archiv eingerichtet. Das Online-Archiv umfasst alle bisherigen Ausgaben der integration. Eine komfortable Suchfunktion ermöglicht es, einzelne Beiträge der letzten 30 Jahre einfach und schnell zu finden.

Alle Artikel sind mit Angaben zu Autorin/Autor, Titel (inkl. englischer Übersetzung), Ausgabe, Seitenzahlen sowie mit Schlagworten versehen. Neuere Beiträge stehen zudem als Abstract sowie als Auszug oder Volltext zur Verfügung. Dadurch werden gezielte Literaturrecherchen einfacher.

Das umfassende Archiv und die Suchfunktion finden Sie ab September unter: www.zeitschrift-integration.de 